\title{
Resonant microwave properties of a voltage-biased single-Cooper-pair transistor
}

\author{
L. Y. Gorelik,, , * S. I. Kulinich,,${ }^{1,2}$ R. I. Shekhter, ${ }^{1}$ and M. Jonson ${ }^{1}$ \\ ${ }^{1}$ Department of Applied Physics, Chalmers University of Technology and Göteborg University, SE-412 96 Göteborg, Sweden \\ ${ }^{2}$ B. I. Verkin Institute for Low Temperature Physics and Engineering, 47 Lenin Avenue, 61103 Kharkov, Ukraine
}

(Dated: November 10, 2018)

\begin{abstract}
We consider the microwave dynamics and transport properties of a voltage-biased single-Cooperpair transistor. The dynamics is shown to be strongly affected by interference between multiple microwave-induced inter-level transitions. As a result the magnitude and direction of the dc Josephson current are extremely sensitive to small variations of the bias voltage and to changes in the frequency of the microwave field.
\end{abstract}

PACS numbers: 72.25.Hg, 73.43.Jn., 73.61.Ey, 72.50.Bb

\section{INTRODUCTION}

It has long been known that transport properties of a superconducting weak link may be strongly affected by external time dependent (ac) fields. To give an example, just after the discovery of the Josephson effect it was found that an alternating rf-field will rectify the ac Josephson current that results from a dc voltage bias and produce voltage steps - Shapiro steps - in the currentvoltage characteristics 1 . This effect can be viewed as a synchronization of the Josephson current oscillations of frequency $\omega_{J}=2 \mathrm{eV} / \hbar$, generated by a bias voltage $V$, and the current oscillations induced by the ac field. If the Josephson coupling energy $E_{J}$ is a simple harmonic function of the superconducting phase difference $\Phi, E_{J}(\Phi)=E_{J} \cos \Phi$, the positions $V^{(n)}$ of the Shapiro steps are given by the expression $\mathrm{eV}^{(n)}=n \hbar \omega$ $(n=1,2, \ldots)$, where $\omega$ is the frequency of the ac field. Deviations from a simple harmonic relation between $E_{J}$ and $\Phi$ result in additional "fractional" steps at voltages $e V^{(n m)}=n \hbar \omega / m$. However their heights are exponentially small when $m \gg n$.

New opportunities to influence the transport properties of Josephson junctions by external ac fields appear in mesoscopic systems, where only few quantum states are responsible for the superconducting charge transfer. For such weak links the Josephson energy - no longer a macroscopic quantity (being proportional to the area of the junction) - may be of the same order of magnitude as the energy quantum $\hbar \omega$ of the external ac field. If so, the alternating field may significantly affect the current by inducing resonant inter-level transitions between charge carrying states in the junction and thereby changing their population. The internal structure of the mesoscopic weak links determines the physical origin of the discrete energy spectrum and the relative positions of the energy levels inside the superconducting gap $2 \Delta$.

Superconducting point contacts (SPCs) and singleCooper-pair transistors (SCPTs) are two fundamentally different examples of mesoscopic weak links. In an SPC such as the one sketched in Fig. 19, the rapid variation of the superconducting phase across the junction gives rise to quasiparticle bound states (Andreev states) that (a)
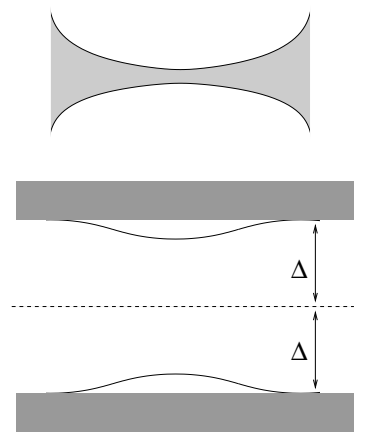

FIG. 1: Schematic energy spectrum of (a) a superconducting point contact (SPC) and (b) a single-Cooper-pair transistor (SCPT). Energies are plotted as a function of the superconducting phase difference $\Phi$ across the system. An important difference between these two examples of a mesoscopic weak link is that the discrete energy levels inside the gap $\Delta$ are well separated from the continuum levels (dark bands) for an SCPT but not for an SPC (see text).

are localized to the vicinity of the contact. The discrete energy levels (Andreev levels) associated with these states appear inside the superconducting gap as shown in Fig. 17. Their positions $\epsilon_{i}$ are defined by the single electron scattering matrix and in the case of low transparency contacts one finds that $\Delta-\left|\epsilon_{i}\right| \ll \Delta$. In an SCPT, which is composed of two low-transparency tunnel junctions in series forming a small island as sketched in Fig. 1b, the Coulomb blockade phenomenon ${ }^{2}$ strongly affects the structure of the energy spectrum. If the superconducting gap $\Delta$ is greater than the Coulomb energy of a grain charged by a single electron, the ground state of the SCPT with a completely isolated central dot, for certain gate voltages, is degenerate with respect to adding one more Cooper pair to the $\operatorname{dot}^{3.4}$. Weak Cooper-pair tunnelling that removes this degeneracy gives rise to current carrying Cooper-pair resonant states, separated as illustrated in Fig. 1b - from the continuum spectrum by an energy of order $\Delta^{5.6 .7 .8 .9}$.

There is a qualitative difference between an SPC and an SCPT in how the energy spectrum depends on the 


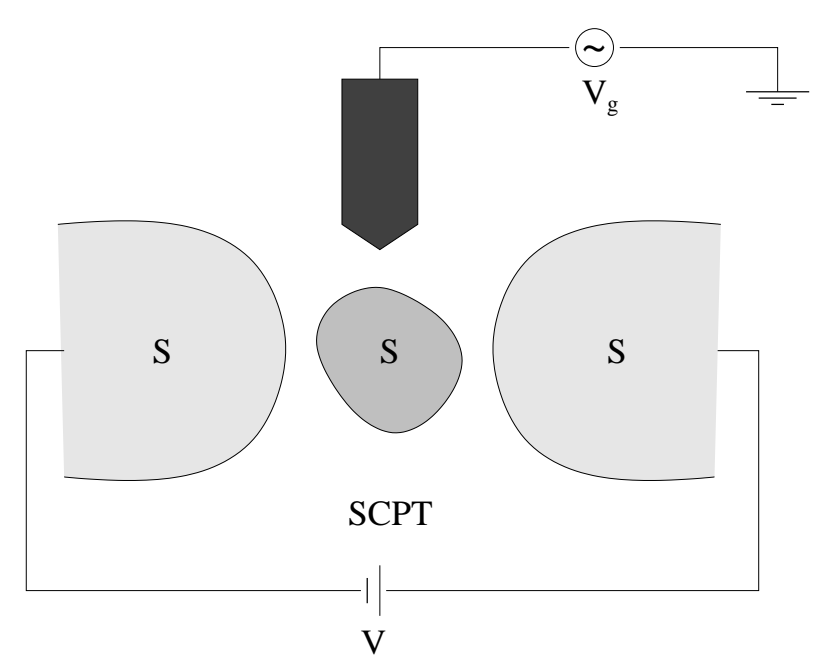

FIG. 2: Sketch of a single-Cooper-pair transistor (SCPT) composed of two low-transparency tunnel junctions in series forming a small superconducting $(\mathrm{S})$ island bridging the gap between two superconducting bulk leads. The potential of the island is controlled by a voltage $V_{g}$ applied to the gate electrode, which is capacitively coupled to the island. A bias voltage $V$ is applied across the SCPT giving rise to a linear time dependence of the phase difference $\Phi=\omega_{J} t \equiv 2 \mathrm{eVt} / \hbar$ between the leads

superconducting phase change across the system. In an SPC the subgap level (as described above this is a discrete energy level in the gap between the ground state and the continuum excitation spectrum) is mixed with the continuum spectrum at certain values of the phase difference, while the Cooper-pair resonant levels in an SCPT are always separated from the continuum, forming a well defined two-level system. The microwave dynamics of a biased SPC was explored in Ref. 10, where it was shown that quantum interference among a sequence of temporally localized inter-level transitions results in nontrivial transport properties of a Josephson weak link of this type. In an SPC, however, coherence may be preserved only during a fixed length of time, since the discrete Andreev states die away as they periodically merge with the continuum spectrum at certain values of the phase difference. (We recall that a voltage bias makes the phase difference increase linearly with time). In contrast, since the discrete energy levels are here always separated from the continuum, the microwave-induced transport properties of a biased SCPT is to a great extent defined by the coherent dynamics of the two-level system and strongly affected by interference processes. In order to demonstrate this phenomenon, we explore below the microwave dynamics and transport properties of a biased SCPT.

\section{MODEL}

A sketch of the SCPT structure to be considered here is presented in Fig. 2] where a nanoscale superconduct- ing island - capacitively coupled to a gate electrode is shown to serve as a bridge between two bulk superconducting leads. The Hamiltonian for the system is

$\hat{H}=\frac{(2 e \hat{n}+Q)^{2}}{2 C}-E_{J}^{L} \cos (\Phi / 2-\hat{\phi})-E_{J}^{R} \cos (\Phi / 2+\hat{\phi})$,

where the Cooper-pair number operator ${ }^{11} \hat{n}$ and the phase operator $\hat{\phi}$ refer to the superconducting condensate on the grain, $[\hat{\phi}, \hat{n}]=i ; E_{J}^{L(R)}$ is the Josephson coupling energy between the grain and the left (right) lead, $C$ is the total mutual capacitance and $Q=C_{g} V_{g}$ is an induced charge controlled by the gate voltage $V_{g}$. The first term in the Hamiltonian governs the electrostatic energy associated with the number of extra Cooper pairs on the grain, while the second and third terms describe the Josephson coupling between grain and leads. Below we consider the symmetric case for which $E_{J}^{L}=E_{J}^{R}=E_{J}$. Therefore, the Josephson part of the Hamiltonian simplifies to $-2 E_{J} \cos (\Phi / 2) \cos \hat{\phi}$.

It is well known that if the characteristic Coulomb energy $E_{q} \sim(2 e)^{2} / 2 C$ associated with charge fluctuations due to Cooper pair exchange between grain and leads, is significantly larger than the Josephson energy $E_{J}$, while at the same time the Coulomb energy $\sim e^{2} / 2 C$ related to single electron fluctuations is smaller than the superconducting energy gap, the low-energy states of the grain are given by a superposition of only two charge states that differ in charge by $2 \mathrm{e}$. Below we will assume that the gate voltage is chosen in such a way that $Q=-e+\delta Q$ where $|\delta Q| \ll e$. In that case the state of the grain is a superposition of the charge state $|0\rangle$, describing the neutral grain, and the charge state $|1\rangle$ with one extra Cooper pair. If $\delta Q=0$ the Coulomb energy in the states $|0\rangle$ and $|1\rangle$ are the same. This degeneracy is, however, removed by the Josephson coupling that induces Cooper pair exchange between the mesoscopic grain and the bulk electrodes. As a result the eigenstates of the Hamiltonian (11) with eigenvalues $E_{ \pm}=\mp E_{J} \cos \Phi / 2$ are given by symmetric and antisymmetric superpositions of the states $|0\rangle$ and $|1\rangle:| \pm\rangle=(|0\rangle \pm|1\rangle) / \sqrt{2}$. These states, which we will refer to as Cooper-pair resonant states, will - if occupied - carry currents given by $j_{ \pm}= \pm e E_{J} \hbar^{-1} \sin \Phi / 2$.

A bias voltage makes the phase difference over the junction change with time, $\Phi(t)=\omega_{J} t+\Phi_{0}$. This time dependence generates a periodic variation of the relative positions of the Josephson levels as well as a periodic change in the current that can be carried by the corresponding resonant Cooper pair states (see Fig. 31). The total Josephson current through the junction depends on the relative "population" $\delta \rho=\rho_{+}-\rho_{-}$of the levels and is given by the formula $j_{J}=e E_{J} \hbar^{-1} \delta \rho \sin \Phi / 2$ ( $\rho_{ \pm}$are probabilities to find the system in the states $| \pm\rangle$, $\left.\rho_{+}+\rho_{-}=1\right)$. One can find important differences between the ac Josephson effect in such a system and in ordinary Josephson junctions. If the Coulomb blockade is totally lifted, there are no inter-level transitions as the levels cross each other and consequently $\delta \rho$ is controlled 


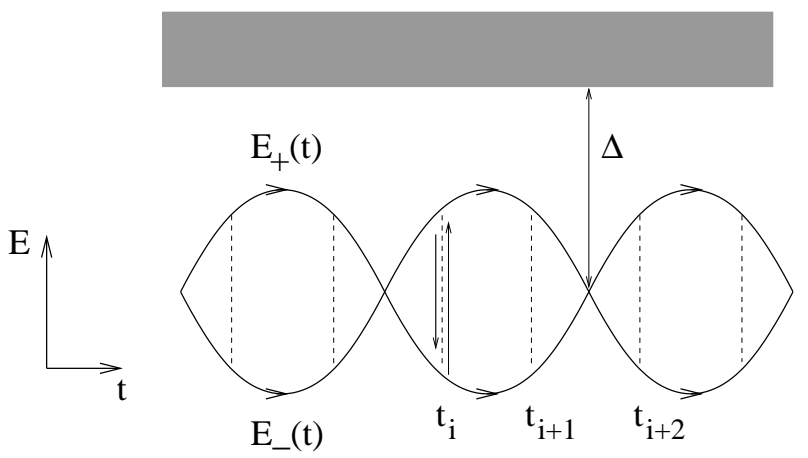

FIG. 3: Time evolution of the Josephson levels $E_{ \pm}(t)$ in the single-Cooper-pair transistor (SCPT) of Fig. 2 Dashed lines represent microwave-induced inter-level transitions occurring at times $t_{i}$, when the resonance condition $\left|E_{+}\left(t_{i}\right)-E_{-}\left(t_{i}\right)\right|=$ $\hbar \omega$ is satisfied.

only by relaxation processes. Even if the temperature is much lower than the characteristic inter-level distance, a weak relaxation to the ground state with rate $\nu \ll \omega_{J}$ leads to an equalization of the level populations. As a result the Josephson current will be proportional to $\nu$ and in the limit $\nu \rightarrow 0$ not only the dc but also the ac current through the Josephson junction vanishes.

The situation is different if an oscillatory voltage is applied to the gate. A time dependent gate voltage makes the induced charge vary in time as well, $\delta Q=$ $\delta Q_{0} \cos (\omega t+\varphi)$. This in turn gives rise to inter-level transitions which control the level populations and by this means one may stimulate the Josephson current. The most interesting case, which will be considered below, appears when the frequency of the gate voltage oscillations $\omega$ is of the same order as the frequency of charge fluctuations on the grain $E_{J} / \hbar$. In this case the interlevel transitions have a resonant nature and significantly influence the Cooper pair transport even for relatively small variations of the gate potential.

In the present work we consider a situation where the energy quantum $\hbar \omega$ of gate voltage oscillations, is of the same order but smaller than the maximum inter-level energy distance $2 E_{J} / \hbar$. At the same time we take $\omega$ to be much larger than the rate of the gate-voltage induced resonant inter-level transitions, $e \delta Q_{0} / \hbar C$. Under these conditions and if one considers the time evolution of the system at low bias voltages, $\omega_{J} \ll \omega$, one can neglect inter-level transitions except during short time intervals $\delta t_{i}$ near the times $t_{i}$, when the resonance condition ${ }^{12}$ $\hbar \omega= \pm 2 E_{J} \sin \Omega t_{i}$ is fulfilled $\left(\Omega=\omega_{J} / 2\right)$. During these time intervals Landau-Zener type inter-level transitions will occur (see Fig. 31). Therefore the evolution of the system we are interested in is given by a sequence of scattering events separated by periods of "free" evolution, during which the level populations do not change.

Despite the fact that the level positions vary periodically in time with period $T_{0}=2 \pi / \Omega$, the dynamics of the system, strictly speaking, is not periodic. This is because the phases of the complex elements of the scattering matrix depend on the phase $\omega t_{i}+\varphi$ which defines the value of induced charge at time $t_{i}$. This fact is of great importance for the transport properties of the system. As we will show below, if the ratio $\omega / \Omega$ is an irrational number the dynamics of the system is qusiperodic and the dc Josephson current is equal to zero. But if this ratio is a rational number, then a dc Josephson current does flow through the junction. The maximum value of the current appears when $\omega / \Omega$ is an integer.

\section{THEORY}

Using the Cooper-pair resonant states representation one can recast the Hamiltonian (10) in the form

$$
\hat{H}(t)=E_{J} \sigma_{3} \sin \Omega t+\varepsilon \sigma_{1} \cos (\omega t+\varphi),
$$

where $\varepsilon=2 e \delta Q_{0} / C$ and $\sigma_{i}$ are the Pauli matrices. As already anticipated, we will assume that the following relations are fulfilled:

$$
\begin{aligned}
& \varepsilon, \hbar \Omega \ll \hbar \omega \leq E_{J} \ll \Delta, \\
& \omega / \Omega=N+p / q, p<q, N \gg 1 .
\end{aligned}
$$

Here $N, p$, and $q$ are integer numbers. The final result, other than details of calculations, does not depend in any essential way on the arithmetic properties of $N, p, q$, and for definiteness we take $q$ to be an even number ( $p$ is odd). Under these conditions the Hamiltonian (2) is a periodic function of time with period $T=q T_{0}$.

The dc Josephson current is given by expression

$$
I=\frac{1}{T} \int_{0}^{T} d t \operatorname{Tr} \hat{\rho}(t) \hat{\jmath}(t)=\frac{2}{T} \int_{0}^{T / 2} d t \operatorname{Tr} \hat{\rho}(t) \hat{\jmath}(t),
$$

where

$$
\hat{\jmath}(t)=\frac{2 e}{\hbar} \frac{\partial \hat{H}(t)}{\partial \Phi}=\frac{e E_{J}}{\hbar} \sigma_{3} \cos \Omega t
$$

is the current operator, $\hat{\rho}(t)$ is the density matrix of the two-level system, while its time evolution is governed by the Liouville-von Neumann equation

$$
\imath \hbar \frac{\partial \hat{\rho}}{\partial t}=[\hat{H}, \hat{\rho}]-\imath \hbar \nu\left(\hat{\rho}-\hat{\rho}_{e q}\right) .
$$

Here $\hat{\rho}_{e q}(t)=\left(1-\sigma_{3} \tanh \left(\beta E_{J} \sin \Omega t\right)\right) / 2$ is the quasistatic density matrix for the unperturbed Hamiltonian $(\varepsilon=0), \beta$ is the inverse temperature, $\nu$ is the relaxation rate. The formal solution of Eq. (5) can be expressed as

$$
\hat{\rho}(t)=\nu \int_{-\infty}^{t} d t^{\prime} e^{\nu\left(t^{\prime}-t\right)} \hat{u}\left(t, t^{\prime}\right) \hat{\rho}_{e q}\left(t^{\prime}\right) \hat{u}^{\dagger}\left(t, t^{\prime}\right),
$$

where

$$
\hat{u}\left(t_{2}, t_{1}\right)=\hat{T} \exp \left[-\frac{\imath}{\hbar} \int_{t_{1}}^{t_{2}} d t \hat{H}(t)\right]
$$


is an evolution operator possessing the symmetry properties

$$
\hat{u}\left(t_{2}+q T_{0} / 2, t_{1}+q T_{0} / 2\right)=\sigma_{3} \hat{u}\left(t_{2}, t_{1}\right) \sigma_{3},
$$

which derives from the symmetry of the Hamiltonian (2),

$$
\hat{H}(t+T / 2)=\sigma_{3} \hat{H}(t) \sigma_{3}
$$

It follows that the density matrix $\rho(t)$ satisfies the relation

$$
\hat{\rho}(t+T / 2)=\sigma_{3} \hat{\rho}(t) \sigma_{3},
$$

which we used in Eq. (4). In the limit $\nu T \ll 1$ this relation together with Eq. (6) immediately results in the equation

$$
\hat{\rho}_{0}=(1-\tilde{\nu}) \hat{U} \hat{\rho}_{0} \hat{U}^{\dagger}+\tilde{\nu} \hat{F}
$$

for the density matrix $\hat{\rho}_{0}=\hat{\rho}(t=0)$. Here $\tilde{\nu}=\nu T / 2 \ll 1$ and

$$
\begin{gathered}
\hat{U}=\sigma_{3} \hat{u}\left(q T_{0} / 2,0\right) \\
\hat{F}=\frac{2}{T} \int_{0}^{T / 2} d t \quad \sigma_{3} \hat{u}(T / 2, t) \hat{\rho}_{e q v}(t) \hat{u}^{\dagger}(T / 2, t) \sigma_{3}
\end{gathered}
$$

Equation (9) and the spectral properties of the unitary operator $\hat{U}$ will play a central role as we proceed.

In the limit $\tilde{\nu}=\nu T / 2 \ll 1$ the solution of Eq.(9) has the form

$$
\hat{\rho}_{0}=\hat{I} / 2+B\left(\left|\lambda_{1}\right\rangle\left\langle\lambda_{1}|-| \lambda_{2}\right\rangle\left\langle\lambda_{2}\right|\right)+\mathcal{O}(\tilde{\nu}),
$$

where $\left|\lambda_{k=1,2}\right\rangle$ are the eigenvectors of the operator $\hat{U}$ with eigenvalues $\exp \left(i \lambda_{k}\right)$ and $B=\left(\left\langle\lambda_{1}|\hat{F}| \lambda_{1}\right\rangle-\left\langle\lambda_{2}|\hat{F}| \lambda_{2}\right\rangle\right) / 2$. From the symmetry of the Hamiltonian (2) it follows that $\sigma_{2} \hat{H}(t) \sigma_{2}=-\hat{H}(t),\left|\lambda_{2}\right\rangle=\sigma_{2}\left|\bar{\lambda}_{1}\right\rangle$ and $\lambda_{1}+\lambda_{2}=\pi$. The expression $\hbar \lambda_{k} / T$ gives the quasienergies of the periodic Hamiltonian, $\hat{H}(t)$; we will refer to $\lambda_{k}$ as a quasienergy factor.

The average Josephson current, Eq.(4), can now be cast in the form

$$
\begin{aligned}
& I=B \sum_{k}(-1)^{k+1}\left\langle\lambda_{k}\left|\hat{I}_{0}\right| \lambda_{k}\right\rangle \\
& \hat{I}_{0}=\frac{2 e E_{J}}{\hbar T} \int_{0}^{T / 2} d t \cos \Omega t \hat{u}^{\dagger}(t, 0) \sigma_{3} \hat{u}(t, 0) .
\end{aligned}
$$

In order to calculate the population coefficient $B$ and the average current in the quasienergy states, $\left\langle\lambda_{k}\left|\hat{I}_{0}\right| \lambda_{k}\right\rangle$, it is convenient to introduce an effective Hamiltonian, $\hat{H}(t ; \xi)$, of the form

$$
\hat{H}(t ; \xi)=\hat{H}(t)+\xi \sigma_{3} \tanh \left(\beta E_{J} \sin \Omega t\right) .
$$

One can show that Eq. (12) for the Josephson current can be expressed as

$$
I=\frac{2 e \hbar \omega}{\pi q T} \lim _{\xi \rightarrow 0} \frac{\partial \lambda_{1}}{\partial \varphi} \frac{\partial \lambda_{1}}{\partial \xi}
$$

where $\lambda_{1}(\varphi ; \xi)$ is the appropriate quasienergy factor of the effective Hamiltonian (14).

Only resonant inter-level transitions are of importance if the microwave interaction energy, $\varepsilon$, is much smaller than the characteristic inter-level distance $2 E_{J} \sim \hbar \omega$. Ignoring the nonresonant part of the Hamiltonian $\hat{H}(t ; \xi)$ (i.e. using the resonance approximation) one finds that the evolution operator $\hat{u}\left((n+1) T_{0} / 2, n T_{0} / 2\right)$ has the form

$$
\begin{aligned}
& \hat{u}\left((n+1) T_{0} / 2, n T_{0} / 2 ; \xi\right)=\sigma_{1}^{n} e^{-i\left((n+1) \omega T_{0} / 2+\varphi\right) \sigma_{3} / 2} \\
& \quad \times \hat{u}_{r e s}\left(T_{0} / 2,0 ; \xi\right) e^{i\left(n \omega T_{0} / 2+\varphi\right) \sigma_{3} / 2} \sigma_{1}^{n}
\end{aligned}
$$

Here $\hat{u}_{\text {res }}\left(T_{0} / 2,0 ; \xi\right)$ is the evolution operator generated by the Hamiltonian $\hat{H}_{r e s}(t ; \xi)$, where

$$
\begin{aligned}
\hat{H}_{r e s}(t ; \xi)=\left(E_{J} \sin \Omega t-\hbar \omega / 2\right. & + \\
\left.\xi \tanh \left(\beta E_{J} \sin \Omega t\right)\right) \sigma_{3} & +\varepsilon \sigma_{1} .
\end{aligned}
$$

From the symmetry of the Hamiltonian $\hat{H}_{r e s}(t ; \xi)$ it follows that the operator $\hat{u}_{\text {res }}\left(T_{0} / 2,0 ; \xi\right)$ has the form

$$
\begin{aligned}
\hat{u}_{r e s}\left(T_{0} / 2,0 ; \xi\right) & = \\
e^{i \theta \sigma_{3}}\left(\sqrt{1-\tau^{2}}+i \tau \sigma_{1}\right) e^{i \theta \sigma_{3}} & \equiv e^{i \theta \sigma_{3}} \sigma_{1} \hat{L} e^{i \theta \sigma_{3}} .
\end{aligned}
$$

The parameter $\tau$ is the resulting probability amplitude for an inter-level transition when the superconducting phase difference changes from $-\pi$ to 0 .

Equation (16) permits us to represent the desired evolution operator $\hat{U}$ of Eq. (10) as

$$
\begin{aligned}
& \hat{U}(\xi)=\sigma_{3} \prod_{n=0}^{q-1} \hat{u}\left((n+1) T_{0} / 2, n T_{0} / 2 ; \xi\right)= \\
& \pm i e^{-i \sigma_{3}(\theta-\varphi / 2)}\left[\prod_{n=0}^{q-1}\left(\hat{L} e^{i(\pi \alpha n+\varphi) \sigma_{3}}\right)\right] e^{i \sigma_{3}(\theta-\varphi / 2)}
\end{aligned}
$$

where $\alpha \equiv p / q$ and the sign $( \pm)$ depend on the arithmetic properties of the natural numbers $N, p$, and $q$.

Now one can see that the eigenvalues of the unitary operator $\hat{U}(\xi)$ can be found by solving the difference equation

$$
|\psi(n+1)\rangle=\hat{L} e^{i(\pi \alpha n+\varphi) \sigma_{3}}|\psi(n)\rangle
$$

with the boundary condition $|\psi(q)\rangle=e^{i \lambda_{k}}|\psi(0)\rangle, k=$ 1,2. A "quasiperiodic" equation of this type may be analyzed by method developed in Ref. 13. The result for the quasienergies $\lambda_{k}$ from such an analysis is

$$
\lambda_{k}=(-1)^{k} \arccos \left(\tau^{q} \cos q \varphi\right)+c_{k}(p, q, N),
$$

where the constants $c_{k}$ are irrelevant.

Taking into account Eqs. (15) and (19) the average current can now be rewritten as

$$
I=-\frac{e \hbar \omega}{2 \pi T_{0}} \frac{\tau^{2(q-1)} \sin 2 q \varphi}{1-\tau^{2 q} \cos ^{2} q \varphi}\left(\frac{\partial \tau^{2}}{\partial \xi}\right)_{\xi=0}
$$


In this work we restrict ourselves to the low temperature limit, where $\beta E_{J} \gg 1$. In this limit $\hat{H}_{\text {res }}(t ; \xi)=$ $\hat{H}_{\text {res }}(t ; \xi-\hbar \omega / 2)$ and, as a consequence, $\partial \tau^{2} / \partial \xi=$ $-2 \hbar^{-1} \partial \tau^{2} / \partial \omega$. Therefore, in order to calculate the current at low temperatures one can use the Hamiltonian $\hat{H}_{\text {res }}(t ; \xi=0)$.

As already mentioned, only resonant inter-level transitions are important here. In the time interval $\left(0, T_{0} / 2\right)$ the resonant condition $2 E_{J} \sin \Omega t_{i}=\hbar \omega$ is fulfilled only at the two times $t_{0}=\Omega^{-1} \arcsin \hbar \omega / 2 E_{J}$ and $t_{1}=$ $T_{0} / 2-t_{0}$. As these "points" in time are reached, interlevel Landau-Zener transitions 14 (see Fig. 3) occur with probability amplitude $w^{1 / 2} e^{i \vartheta}$, where

$$
w=1-\exp \left[-\frac{\pi \varepsilon^{2}}{4 E_{J} \hbar \Omega}\left[1-\left(\frac{\hbar \omega}{2 E_{J}}\right)^{2}\right]^{-\frac{1}{2}}\right] .
$$

Dividing time interval $\left(0, T_{0} / 2\right)$ into periods of free evolution (where interactions with the microwave field can be neglected) and describing the passage through the resonant points by a Landau-Zener scattering matrix, one finds the probability for an inter-level transition to be

$$
\begin{aligned}
& \tau=2 w \sqrt{1-w^{2}} \cos (\Theta+\vartheta) \\
& \Theta=\hbar^{-1} \int_{t_{0}}^{T_{0} / 2-t_{0}} d t E_{J} \sin \Omega t-\omega\left(T_{0} / 4-t_{0}\right)
\end{aligned}
$$

and the final expression for the microwave-induced dc Josephson current becomes

$$
I=\frac{e \omega}{\pi^{2}}\left(\arccos \frac{\hbar \omega}{2 E_{J}}\right) \frac{\tau^{2 q} \tan (\Theta+\vartheta)}{1-\tau^{2 q} \cos ^{2} q \varphi} \sin 2 q \varphi .
$$

Equation (21) determines the current-voltage characteristics of the SCPT studied, which depend crucially on the frequency of the microwave irradiation. Its validity is restricted to the special values of $\omega$ and $V$ determined by Eq. (3)

Averaging the current over a long time $T$ (corresponding to a large value of $q$, since $T=q T_{0}$ ), and plotting it as a function of the parameter $\omega / \Omega$, results in many sharp features. (Notice that two values of $\omega / \Omega$ which are arbitrarily close to each other might correspond to widely different values of $q$ in Eq.(3)). It is clear, however, that any upper limit $T_{\max }$ for the allowed averaging time will tend to smooth out the sharp features. The value of $T_{\max }$ might be determined by either a relaxation time or a finite observation time. In either case currents calculated for two values of $(\omega / \Omega)_{1,2}$ will be indistinguishable if $\left|q_{1}-q_{2}\right|>\Omega T_{\max }$. This argument provides a justification for smoothening the current as given by Eq. (21) in order to get a regular dependence $I=I(\omega / \Omega)$. An appropriate procedure is to evaluate Eq. (21) at all rational points for which $q<q_{\max }$. Then a smooth dependence $I(\omega / \Omega)$ can be obtained by making use of an interpolation procedure. The result of such an interpolation procedure is presented in Fig. [4

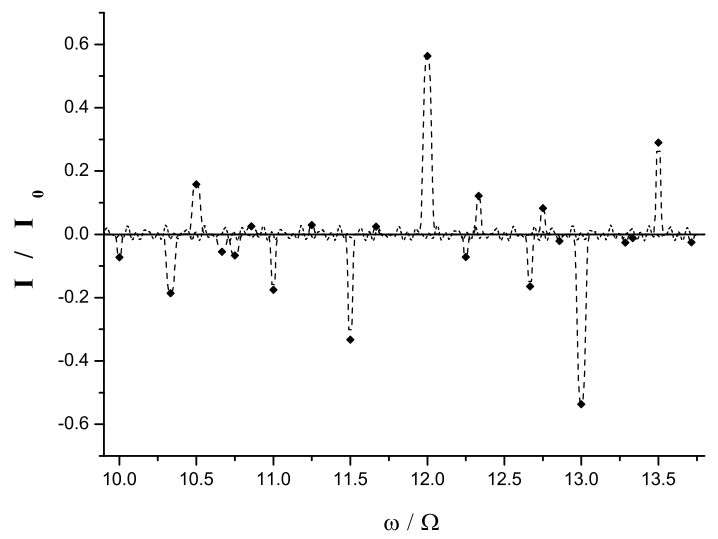

FIG. 4: Microwave-induced current $I$ in units of $I_{0} \equiv e \omega / \pi^{2}$, plotted as a function the microwave frequency $\omega$ normalized to $\Omega \equiv \omega_{J} / 2$. The result was obtained with $\varphi=$ $\pi / 11, \hbar \omega / 2 E_{J}=0.001, w=0,5$ and $q_{\max }=7$ (see text).

\section{CONCLUSION}

We have shown that the microwave dynamics of a single-Cooper-pair transistor is strongly influenced by interference effects caused by the quantum dynamics of the superconducting dot, which — as in Fig. 2 - forms the central island in the transistor structure. The sharp peaks that were found in the current-voltage $(I-V)$ characteristics for "fractional" values of the voltage, as shown in Fig. 4 are a signature of a resonant interaction with the microwave field. The peaks occur when $\mathrm{eV}=\hbar \omega /(N+p / q)$, where $N, p$, and $q$ are integers; they are finite if $N \rightarrow \infty$ and do not vanish in the limit of weak microwave irradiation, The interaction with the microwave field results in multiple, coherent transitions between resonant Cooper-pair levels. This picture, which differs qualitatively from the Shapiro effect $\frac{1}{\underline{t}}$, is a direct manifestation of the role of strong Coulomb correlations in the nonequilibrium superconducting dynamics of mesoscopic weak links. The Coulomb blockade of Cooper pair tunnelling reduces the number of relevant charge states to two, and is responsible for the ensuing quantum coherent two-level dynamics of Cooper pair resonant states.

A single-Cooper-pair transistor requires the Coulomb blockade energy $E_{q}$ to be much larger than the Josephson coupling energy $E_{J}$. If instead $E_{J} \gg E_{q}$, a large number of dot charge states with different number of Cooper pairs are degenerate. As a result tunnel coupling to the leads form dot states with a well defined superconducting phase. Microwave properties in this limit are governed by the classical dynamics of the superconductiong phases, caused by the driving voltage and the microvawe field. If we were to imagine that $E_{q}$ could be diminished from a value much larger than $E_{J}$ to a value much smaller than $E_{J}$, we would witness a transition from a situation where 
the quantum dynamics of the superconducting phase was relevant to one where the classical dynamics would dominate. During such a transition the resonance microwave absorption would disappear and be replaced by standard Shapiro signatures, known from macroscopic weak links.
Research Council, VR, (LYG, RIS). SIK acknowledges the hospitality of the Department of Applied Physics at Chalmers University of Technology and Göteborg University.

\section{Acknowledgments}

This work has been supported financially by the Royal Swedish Academy of Sciences, KVA, and by the Swedish

* Electronic address: gorelik@fy.chalmers.se

1 S. Shapiro, Phys. Rev. Lett. 11, 80 (1963).

2 I. O. Kulik and R. I. Shekhter, Sov. Phys. JETP, 41, 308 (1975).

3 K. A. Matveev, M. Gisselfält, L. I. Glazman M. Jonson and R. I. Shekhter, Phys. Rev. Lett. 70, 2940 (1993).

${ }^{4}$ F. W. J. Hekking, L. I. Glazman, K. A. Matveev and R. I. Shekhter, Phys. Rev. Lett. 70, 4138 (1993).

5 Y. Nakamura, C. D. Chen, J. S. Tsai, Phys. Rev. Lett. 79, 2328 (1997).

${ }^{6}$ Y. Nakamura, Yu. A. Pashkin and J. S. Tsai, Nature 398, 786 (1999); Y. Nakamura and J. S. Tsai, J. Low Temp. Phys. 118, 765 (2000).

7 A. Cottet, D. Vion, A. Aassime, P. Joyez, D. Esteve and M. H. Devoret, Physica C 367, 197 (2002).

${ }^{8}$ L. Y. Gorelik, A. Isacsson, Y. M. Galperin, R. I. Shekhter and M. Jonson, Nature 411, 454 (2001).

9 J. R. Friedman and D. V. Averin, Phys. Rev. Lett. 88, 050403 (2002).

10 L. Y. Gorelik, N. I. Lundin, V. S. Shumeiko, R. I. Shekhter and M. Jonson, Phys. Rev. Lett. 81, 2538 (1998).

11 We count the excess Cooper pairs relative to the neutral state. Hence the eigenstate of the operator $\hat{n}$ with eigenvalue $n=0$ corresponds to a neutral grain.

12 For the sake of definiteness we let $\Phi_{0}=-\pi$.

13 L. Y. Gorelik, S. I. Kulinich, Y. M. Galperin, R. I. Shekhter and M. Jonson, Phys. Rev. Lett. 78, 2196 (1997).

14 L. D. Landau, Phys. Z. Sov. 2, 46 (1932); C. Zener, Proc. Roy. Soc. (London) A 137, 696 (1932); K. Mullen, E. BenJacob, Y. Gefen and Z. Schuss, Phys. Rev. Lett. 62, 2543 (1989). 